\title{
Taylor-Couette flow of shear-thinning fluids
}

F

Cite as: Phys. Fluids 31, 053102 (2019); https://doi.org/10.1063/1.5088143

Submitted: 08 January 2019 . Accepted: 02 May 2019. Published Online: 29 May 2019

N. Cagney (iD), and S. Balabani (iD)

COLLECTIONS

F This paper was selected as Featured
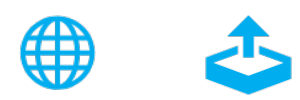

\section{ARTICLES YOU MAY BE INTERESTED IN}

Linear stability analysis of a surfactant-laden shear-imposed falling film

Physics of Fluids 31, 054103 (2019); https://doi.org/10.1063/1.5093745

Geometric optimization of riblet-textured surfaces for drag reduction in laminar boundary layer flows

Physics of Fluids 31, 053601 (2019); https://doi.org/10.1063/1.5090881

Electric field mediated squeezing to bending transitions of interfacial instabilities for digitization and mixing of two-phase microflows

Physics of Fluids 31, 052005 (2019); https://doi.org/10.1063/1.5092198

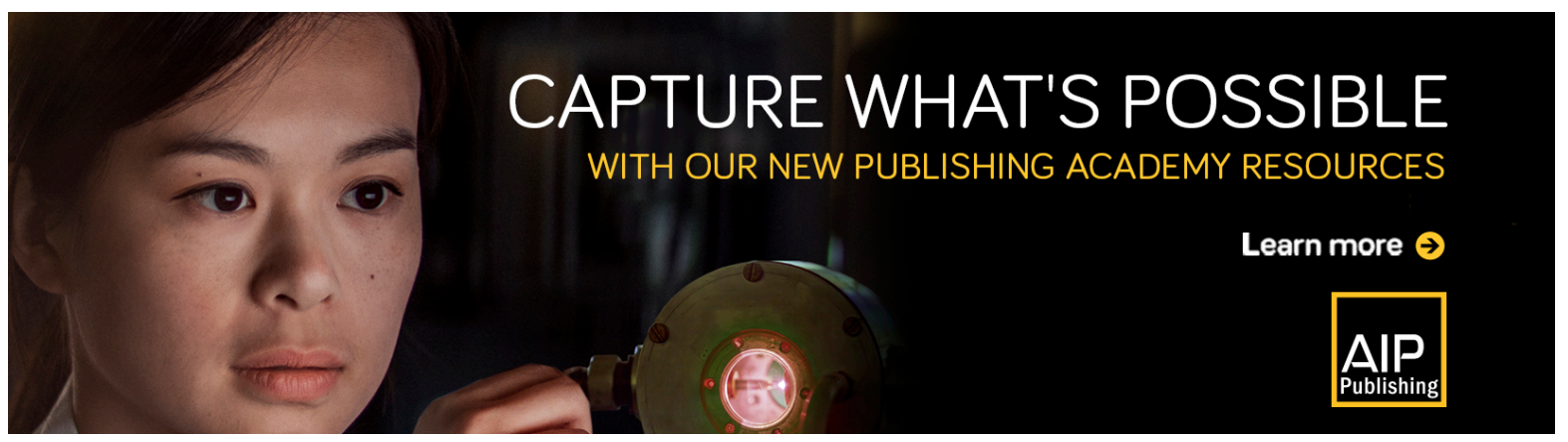




\title{
Taylor-Couette flow of shear-thinning fluids
}

\author{
Cite as: Phys. Fluids 31, 053102 (2019); doi: 10.1063/1.5088143 \\ Submitted: 8 January 2019 • Accepted: 2 May 2019 • \\ Published Online: 29 May 2019
}

\section{N. Cagney ${ }^{1,2}$ (D) and S. Balabani}

\author{
AFFILIATIONS \\ ${ }^{1}$ Department of Mechanical Engineering, University College London, London WC1E 6BT, United Kingdom \\ ${ }^{2}$ School of Engineering and Materials Science, Queen Mary University of London, London El 4NS, United Kingdom
}

\begin{abstract}
The flow between two concentric cylinders, one of which is rotating (Taylor-Couette flow), has been the focus of extensive research, due to the number of flow instabilities that may occur and its use in various industrial applications. We examine Taylor-Couette flow of Newtonian and shear-thinning fluids (solutions of xanthan gum in water/glycerol) using a combination of particle-image velocimetry and flow visualization for a wide range of Reynolds number, spanning the circular Couette flow, Taylor vortex flow, and wavy vortex flow regimes. Shear thinning is associated with an increase in the axial wavelength and has a nonmonotonic effect on the critical Reynolds number for transition to Taylor vortex flow and wavy vortex flow. The magnitude of vorticity and the strength of the radial jets transporting fluid away from the inner cylinder ("outward jets") are both reduced in shear-thinning fluids relative to the Newtonian case; the vorticity in the shear-thinning fluids also tends to concentrate at the edges of vortices, rather than in the cores. In the wavy vortex flow regime for Newtonian fluids, the amplitudes of the waves at the "inward jets" (moving toward the inner cylinder) are low compared to those at the outward jets. However, for the shear-thinning fluids, the amplitudes of the waves at both the inward and outward jets tend to be significantly larger. Finally, shear thinning is associated with greater variations in time and space: we observe slow drifts in the axial positions of vortices and spatial variations in the amplitudes of the wavy instability, which are absent in Newtonian fluids.
\end{abstract}

Published under license by AIP Publishing. https://doi.org/10.1063/1.5088143

\section{INTRODUCTION}

The flow between two concentric cylinders in which one or both are rotating (Taylor-Couette flow) has been the focus of considerable attention for almost a century. ${ }^{1,2}$ Despite the apparent simplicity of the system, it can lead to surprisingly complex dynamics, including numerous instabilities, flow transitions, and chaos. ${ }^{3}$ Considering the most common form of Taylor-Couette flow, in which the inner cylinder is rotating and the outer cylinder is fixed, the dynamics are controlled by the Reynolds number

$$
R e=\frac{\rho \Omega r_{i} d}{\mu},
$$

where $\rho$ is the fluid density, $\mu$ is the viscosity, $\Omega$ is the rotational speed of the inner cylinder, $r_{i}$ is the inner cylinder radius, and $d$ is the gap between the cylinders. The Taylor-Couette system can be geometrically characterized by the radius ratio, $\eta=r_{i} / r_{o}$, where $r_{o}$ is the outer cylinder radius, and the aspect ratio, $A R=d / L$, where $L$ is the length of the cylinder axis.

At low $R e$, the flow is laminar and axisymmetric, and is referred to as Circular Couette Flow (CCF). As the Reynolds number is increased beyond a critical value, $R e_{c, 1}$, the flow becomes unstable and is dominated by a series of steady toroidal vortices. This condition is known as Taylor Vortex Flow (TVF). A second critical Reynolds number exists, $R e_{c, 2}$, at which the flow becomes unsteady and loses its axisymmetry, as azimuthal waves travel through the fluid causing oscillations in the position of vortices. ${ }^{4,5}$ This instability is thought to be driven by jets between vortices which transport fluid at the inner cylinder surface with high angular velocity into the center of the fluid domain, resulting in a strong azimuthal jet. ${ }^{6,7}$ This state, known as Wavy Vortex Flow (WVF), is characterized by a single temporal frequency and wavelength. Further increases in $R e$ are associated with more transitions in which more temporal frequencies appear, ultimately leading to turbulence. ${ }^{1,3,8}$

As well as being of interest from a purely theoretical standpoint, Taylor-Couette flow has been used in many industrial applications, including filtration, ${ }^{9}$ protein shearing, ${ }^{10}$ blood detoxification, ${ }^{11}$ liquid-liquid extraction, ${ }^{12}$ and bioreactors. ${ }^{13-15}$ Many industrial applications of Taylor-Couette flow involve nonNewtonian fluids, e.g., suspensions of cells in bioreactors, blood in detoxification devices, polymers in chemical reactors, or multiphase 
mixtures in extraction systems. In terms of the study of complex fluids, Taylor-Couette flow of non-Newtonian fluids represents an ideal case in which to investigate the role of rheology in flow instabilities. ${ }^{16}$

A common feature of many complex fluids is shear-thinning behavior, which is observed in suspensions, ${ }^{17}$ yield stress fluids, ${ }^{18}$ fluids containing microswimmers, ${ }^{19}$ and many viscoelastic fluids. ${ }^{20}$ The majority of studies in the literature examining Taylor-Couette flow of inelastic shear-thinning fluids have been theoretical or numerical and have focused on the effect of shear thinning on $R e_{c, 1}$. In the case of non-Newtonian fluids in Taylor-Couette flow, the Reynolds number is typically defined using the viscosity that would be observed at a strain rate equal to the average or "nominal" strain rate across the gap, i.e.,

$$
\dot{\gamma}_{\text {nom }}=\frac{\Omega r_{i}}{d} .
$$

Ashrafi $^{16}$ and Coronado-Matutti, Souza Mendes, and Carvalho $^{21}$ predicted that shear thinning lowered the critical Reynolds number for TVF with respect to Newtonian fluids, which was supported by the numerical work of Lockett, Richardson, and Worraker, ${ }^{22}$ Khali, Nebbali, and Bouhadef ${ }^{23}$ and Alibenyahia et al. ${ }^{24}$

Once instabilities set in, the shear-thinning rheology can have a significant effect on the flow. Alibenyahia et al. ${ }^{24}$ predicted the wavelength for a range of radius ratios and flow indices, $n$ (where $n$ is a measure of the shear thinning, with $\mu \sim \dot{\gamma}^{n-1}$ and $n=1$ for Newtonian fluids). For narrow gaps ( $\eta \gtrsim 0.66)$, shear thinning was found to increase the wavelength, while for large gaps $(\eta \lesssim 0.5)$, the opposite trend was predicted. This is broadly consistent with the finite element study of Lockett, Richardson, and Worraker, ${ }^{22}$ who found that for narrow gaps $(\eta=0.95)$, the wavelength increased with decreasing flow index, appearing to reach a maximum at $n \approx 0.1$. At larger gaps $(\eta=0.5)$, the wavelength increased slightly for $n>0.5$, but fell below its Newtonian value as $n$ was reduced.

Several researchers have commented on the lack of experimental data on Taylor-Couette flow of inelastic shear-thinning fluids. ${ }^{16,21,24,25}$ Sinevic, Kuboi, and Nienow ${ }^{26}$ measured the torque acting on the inner cylinder for a Newtonian and three shear-thinning fluids ( $n=0.4,0.5$, and 0.57 ), and estimated the transition to TVF based on changes in the scaling relationship between torque and $R e$. They found that for $\eta=0.7$, shear thinning increased $R e_{c, 1}$, but the effect was not monotonic for $\eta=0.9$, where $R e_{c, 1}$ was reduced for $n=0.5$ and 0.57 , but was slightly higher than the Newtonian value for $n=0.4$. Bahrani et al. ${ }^{27}$ presented measurements for three fluids (one Newtonian and two shear thinning) with a very large gap $\eta=0.4$, and also found that the shear thinning increased the wavelength and had a nonmonotonic effect on $R e_{c, 1}$, in agreement with their predictions from linear stability theory. Finally, Escudier, Gouldson, and Jones ${ }^{25}$ studied the flow field in a Taylor-Couette reactor with a shear-thinning fluid $(n=0.437, \eta=0.506)$ using laser-Doppler anemometry and noted an increase in the asymmetry between the strengths of the inward and outward jets between vortices relative to the Newtonian case, as well as a tendency for the position of vortices to slowly drift in the axial direction, which was not observed for the Newtonian fluid.

It is clear that despite its relevance to numerous industrial applications, there is a lack of experimental data on Taylor-Couette flow with shear-thinning fluids, especially in narrow gaps. It remains unclear what effect shear thinning will have on the flow field and the wavy flow instability. This study aims to address these issues, by providing experimental measurements of the flow field and transitions for Newtonian and shear-thinning fluids over a wide range of $R e$ using a combination of particle-image velocimetry (PIV) and flowvisualization measurements. The remainder of the paper is structured as follows: the experimental details of the Taylor-Couette flow cell and flow measurements are provided in Sec. II, along with details of the rheology of the fluids that are examined; the time-averaged characteristics of the velocity fields are examined in Sec. III A; the transitions and the wavy instability are examined in Sec. III B; low frequency fluctuations in the vortex positions in shear-thinning fluids are discussed in Sec. III C; and finally, some concluding remarks are made in Sec. IV.

\section{EXPERIMENTAL DETAILS}

Experiments were performed in a Taylor-Couette flow cell which has previously been described by Dusting and Balabani ${ }^{28}$ and Imomoh, Dusting, and Balabani. ${ }^{29}$ The test section consisted of a thin-walled, precision bore glass cylinder, mounted vertically between two acrylic plates, and a Teflon inner cylinder that was spray-painted black, to reduce any reflections. The inner and outer cylinders had radii of $r_{i}=21.2 \mathrm{~mm}$ and $r_{o}=25.5 \mathrm{~mm}$, respectively, and the cylinder length was $L=55.5 \mathrm{~mm}$, which corresponded to a gap of $4.3 \mathrm{~mm}$, a radius ratio of $\eta=0.83$ and an aspect ratio of $A R=12.97$. The test section was enclosed within a square chamber, containing the same fluid as in the flow cell, in order to reduce any diffraction effects due to the curvature of the thin outer cylinder.

The inner cylinder was mounted directly onto the drive shaft of a stepper motor (SmartDrive Ltd.). The rotation of the motor

TABLE I. Properties of the fluids examined, including water:glycerol volume ratio, concentration of xanthan gum, and rheological properties of data fit to the Carreau model [Eq. (3)].

\begin{tabular}{lcccccc}
\hline \hline Fluid & $\begin{array}{c}\text { Water:glycerol } \\
\text { volume ratio }\end{array}$ & $\begin{array}{c}\text { Xanthan } \\
\text { concentration }(\mathrm{g} / \mathrm{L})\end{array}$ & $\mu_{0}(\mathrm{mPa} \mathrm{s})$ & $\mu_{\infty}(\mathrm{mPa} \mathrm{s})$ & $\lambda_{c}(\mathrm{~s})$ & $n$ \\
\hline 1 & $3: 1$ & 0 & 2.2 & 2.2 & $\mathrm{n} / \mathrm{a}$ & 1 \\
2 & $4.08: 1$ & 0.291 & 13.64 & 1.732 & 0.5 & 0.68 \\
3 & $1: 0$ & 0.507 & 23.11 & 0.976 & 0.89 & 0.62 \\
4 & $1: 0$ & 0.843 & 85.32 & 0.935 & 1.29 & 0.52 \\
\hline \hline
\end{tabular}




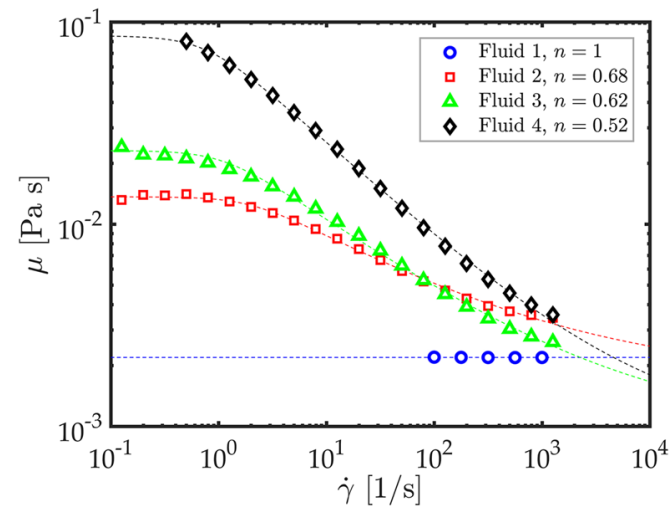

FIG. 1. Flow curves found for the four fluids examined. The lines represent the Carreau model [Eq. (3)], with the various fitting parameters listed in Table I.

was monitored using an optical shaft encoder with a resolution of 2000 pulses per revolution, such that the acceleration rate could be controlled to a high degree of certainty. ${ }^{29}$ The temperature was measured in the reactor at the start and end of every experiment, and the temperature fluctuations were found to be less than $\pm 1.2^{\circ} \mathrm{C}$, which corresponded to a change in viscosity of less than $\pm 2.8 \%$.

The fluids examined consisted of mixtures of distilled water, glycerol, and xanthan gum (Sigma-Aldrich), as listed in Table I. A high-shear mixer (Silverson) was used to ensure that the xanthan gum was fully dispersed within the fluids. The shear rheology of each fluid was measured using a rotational ARES rheometer (TA Instruments), at room temperature, and temperatures slightly above and below in order to assess the effects of small temperature fluctuations during experiments.

The flow curves at room temperature for each fluid are shown in Fig. 1. The rheology could be described using the Carreau model, which is given by

$$
\mu(\dot{\gamma})=\mu_{\infty}+\left(\mu_{0}-\mu_{\infty}\right)\left(1+\left(\lambda_{c} \dot{\gamma}\right)^{2}\right)^{\frac{n-1}{2}},
$$

where $\mu_{0}$ and $\mu_{\infty}$ are the viscosities at zero and infinite shear rate, respectively, $\lambda_{c}$ is the relaxation time and $n$ is the flow index. The dashed lines in Fig. 1 show the best fit of Eq. (3) to the data, and fitting parameters are listed in Table I. As in previous studies in the literature, the Reynolds number was defined based on the viscosity found from the Carreau model and the values in Table I and the nominal strain rate across the fluid gap [Eq. (2)].

Shear-thinning fluids often exhibit some degree of viscoelasticity, although this is often ignored in experimental studies. In order to assess the potential role of viscoelasticity in our experiments, dynamic rheology measurements were acquired for each of the xanthan solutions at a maximum strain rate of $10 \%$ for a range of frequencies, and the storage and loss moduli were measured. The storage modulus, $G^{\prime}$, is the component of the complex viscosity in phase with the strain (i.e., elasticity) and the loss modulus, $G^{\prime \prime}$, is the component in phase with the strain rate (i.e., the shear viscosity). The viscoelasticity of a material can therefore be expressed as tan $\delta=G^{\prime \prime} / G^{\prime}$, where $\delta=90^{\circ}$ for a purely viscous fluid, and $\delta \lesssim 45^{\circ}$ for a fluid in which elastic effects dominate over viscous effects.

The variations in the storage and loss moduli and $\tan \delta$ for each of the fluids tested are shown in Fig. 2. There is a tendency for $\tan \delta$ to reduce with increasing frequency; however, for all frequencies examined, $\tan \delta>45^{\circ}$, indicating that the fluid rheology is dominated by viscous effects. The oscillation frequency, $\omega_{c}$, at which $\delta=45^{\circ}$ can be used to identify the relaxation time, $\lambda=2 \pi / \omega_{c}$. Figure 2(b) shows that this does not occur within the operational range of the rheometer, indicating that $\lambda<6.28 \mathrm{~ms}$.

\section{A. Experimental protocol}

The experiments were performed by slowly increasing the rotational speed of the inner cylinder at a fixed rate. The flow
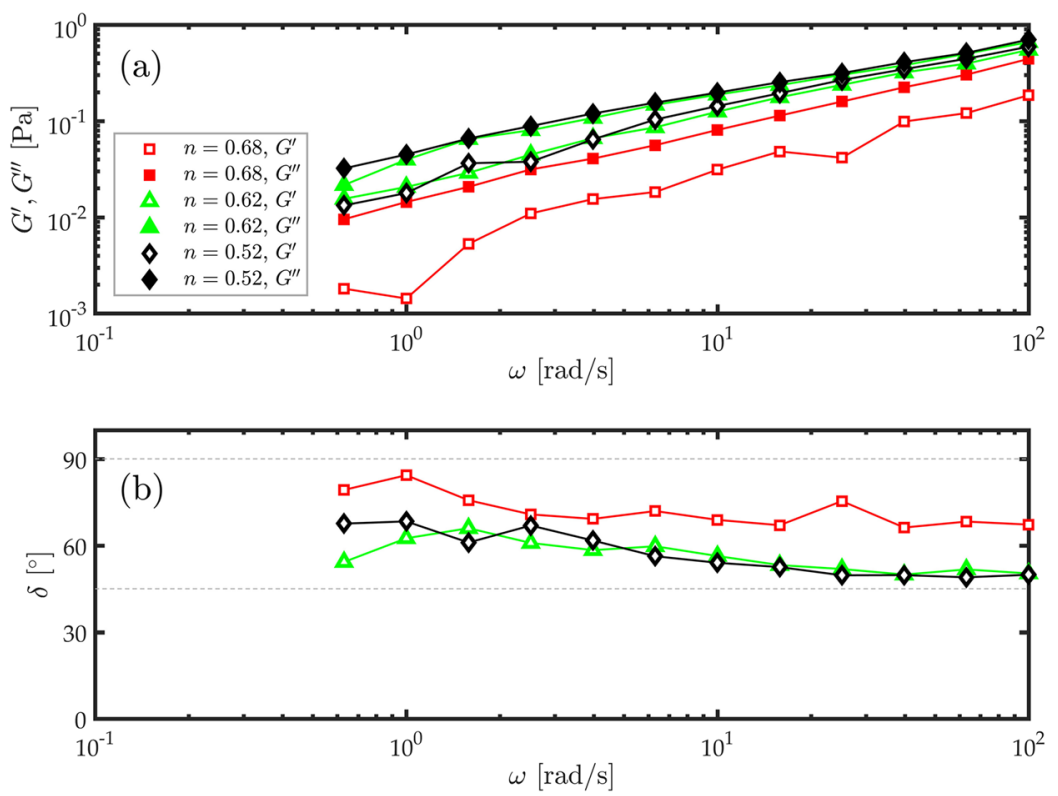

FIG. 2. Storage and loss moduli ( $G^{\prime}$ and $G^{\prime \prime}$, respectively) (a) and $\tan \delta$ (b) for the xanthan solutions examined. 
TABLE II. Experimental conditions used in the various experiments. "PIV" and "vis" denote particle-image velocimetry and flow visualization experiments, respectively, and $f_{s}$ denotes the sampling frequency.

\begin{tabular}{lcccccc}
\hline \hline Fluid & $\begin{array}{c}\Omega_{\text {max }} \\
(\mathrm{rad} / \mathrm{s})\end{array}$ & $\begin{array}{c}\mathrm{d} \Omega / \mathrm{d} t \\
\left(\mathrm{rad} / \mathrm{s}^{2}\right)\end{array}$ & $\begin{array}{c}f_{s}(\mathrm{PIV}) \\
(\mathrm{Hz})\end{array}$ & $\begin{array}{c}\text { Resolution }(\mathrm{PIV}) \\
(\text { Pixels/mm) }\end{array}$ & $\begin{array}{c}f_{s}(\mathrm{vis}) \\
(\mathrm{Hz})\end{array}$ & $\begin{array}{c}\text { Image size } \\
(\text { vis) (Pixels) }\end{array}$ \\
\hline 1 & 22.9 & 0.013 & 40 & 44.8 & 10 & $2560 \times 80$ \\
2 & 45.9 & 0.025 & 80 & 44.5 & 20 & $2560 \times 40$ \\
3 & 39.0 & 0.019 & 80 & 45.8 & 18 & $2560 \times 40$ \\
4 & 61.3 & 0.031 & 80 & 44.8 & 25 & $2560 \times 16$ \\
\hline \hline
\end{tabular}

dynamics are known to be sensitive to the choice of acceleration rate: Dutcher and Muller ${ }^{8}$ argued that the nondimensional acceleration rate, $\mathrm{d} R e / \mathrm{d} t^{*}$ (where $t^{*}=t \mu / \rho d^{2}$ is time divided by the viscous time scale), should be less than unity to ensure that the flow can be assumed to be "quasisteady." In contrast, Xiao, Lim, and Chew ${ }^{30}$ varied $\mathrm{d} R e / \mathrm{d} t^{*}$ over several orders of magnitude and argued that for $\mathrm{d} R e / \mathrm{d} t^{*} \leq 11.23$, the flow state for $R e / R e_{c, 1}<10$ showed no dependence on the acceleration rate.

For non-Newtonian fluids, our definition of the Reynolds number means that $R e$ and $\mathrm{d} R e / \mathrm{d} t^{*}$ vary nonlinearly with $\Omega$ and $\mathrm{d} \Omega / \mathrm{d} t$, respectively. We employ acceleration rates such that for all $R e$ examined, $\mathrm{d} R e / \mathrm{d} t^{*}<5.5$. This represents a balance between the criterion of Dutcher and Muller ${ }^{8}$ and the practical consideration that the longer an experiment takes to perform, the more susceptible it is to temperature changes due to viscous heating that will alter the viscosity and Reynolds number. The acceleration rates for each experiment are listed in Table II.

As the cylinder is slowly accelerated, the velocity field in the meridian plane is measured using particle-image velocimetry (PIV) at irregular intervals. The flow was seeded with rhodamine fluorescent particles with a mean diameter of $10 \mu \mathrm{m}$ (Dantec Dynamics), the meridian $(r-z)$ plane was illuminated using a $532 \mathrm{~nm} \mathrm{Nd:Yag}$ laser, and images were acquired using a Phantom Miro 340 camera, as indicated in Fig. 3. Prior to each experiment, the inner cylinder was rotated at a high speed for a short period of time to ensure that the particles were evenly distributed throughout the fluid. The sampling rate for each experiment is listed in Table II. For each set of PIV measurements, 1024 image pairs were acquired. The image pairs were analyzed using a cross correlation scheme with $50 \%$ overlap and a final vector spacing of $16 \times 16$ pixels. Vectors acquired at $r / d>0.8$ were ignored, in order to prevent the introduction of errors due to the curvature of the test section.

The PIV measurements represented high-temporal resolution images of the velocity over a short period of time. However, in order to examine flow transitions and study how flow properties varied as the Reynolds number was slowly increased, it was necessary to employ an alternative approach that is capable of continuously monitoring the flow. Following the PIV measurements, a small quantity of anisotropic tracers (flakes of mica) was added to the flow, such that changes in the direction of flow could be determined from changes in the amount of light scattered. ${ }^{31}$ The inner cylinder was rotated using an identical protocol to the PIV measurements, the test section was illuminated using a white light-emitting diode (LED) (SugarCUBE, Edmund Optics) and images were acquired of a narrow strip of the test section using the same high-speed camera, as shown in Fig. 3. The images were averaged to form a single column of pixels, and the columns of pixels found from consecutive images were joined to form a spatial-temporal map, following a similar approach to many previous studies of TaylorCouette flow, e.g., Dutcher and Muller, ${ }^{8}$ Bahrani et al., ${ }^{27}$ Abcha et al., ${ }^{31}$ Martínez-Arias et al., ${ }^{32}$ and Majji, Banerjee, and Morris. ${ }^{33}$ The volume fraction of the flakes was very small, $<10^{-4}$, such that they had a negligible effect on the fluid rheology, which was confirmed by means of rheological measurements. Details of the flow visualization measurements performed for each fluid are shown in Table II.
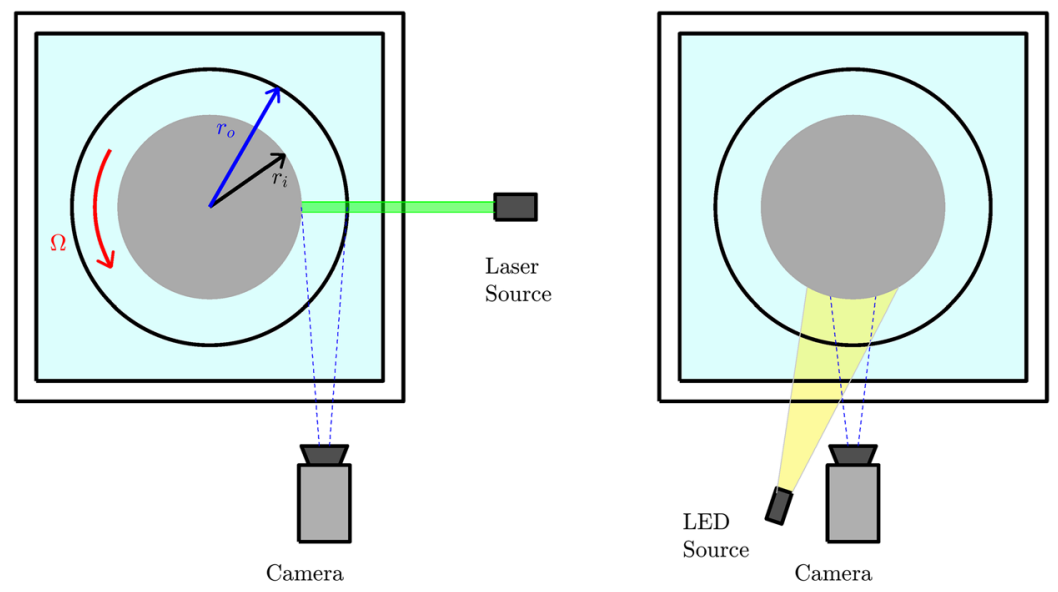

FIG. 3. Plan view of the test section used, showing the PIV measurements (left) and the flow-visualization measurements (right). 

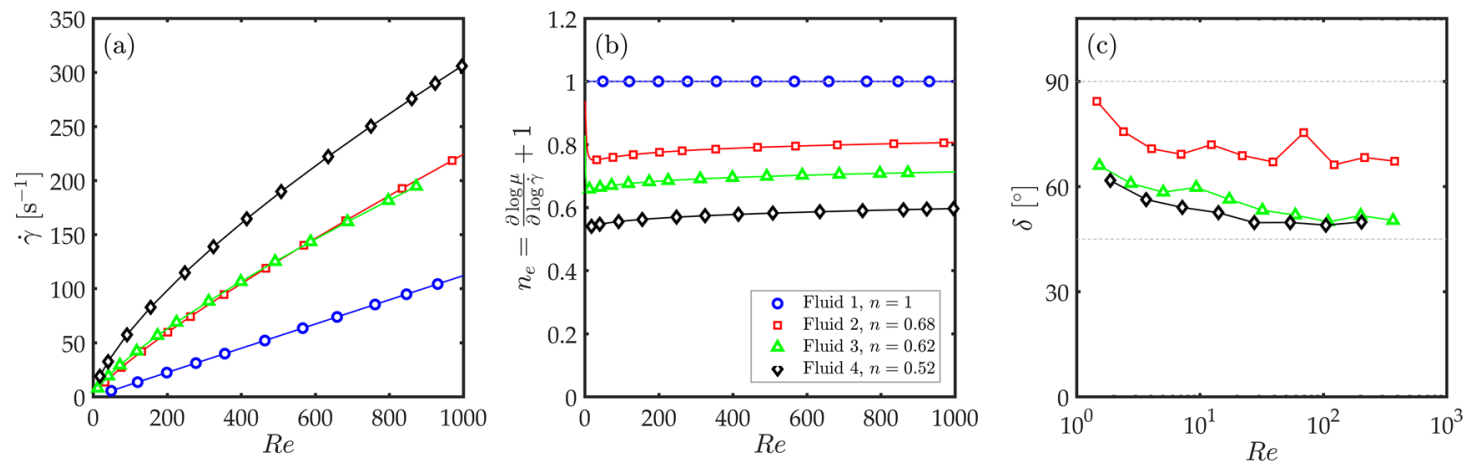

FIG. 4. Variation in strain rates (a), effective flow index [Eq. (4)] (b) and angle $\delta$ (c) as a function of Reynolds number, for each of the fluids examined.

The nominal strain rates encountered in each experiment are presented in Fig. 4(a) as a function of Re, and occur within the range $\dot{\gamma}=0-350$. The flow index in the Carreau model [Eq. (3)] gives a "global" relationship between the viscosity and strain rate, but does not indicate the actual "local" scaling at a given strain rate. This can be found by the "effective" flow index, ${ }^{21}$ which is given by

$$
n_{e}(\dot{\gamma})=\frac{\partial \log \mu}{\partial \log \dot{\gamma}}+1,
$$

where $\mu$ is found using Eq. (3) for any strain rate.

The variation in $n_{e}$ with $R e$ for each experiment is shown in Fig. 4(b). The effective flow index is approximately constant throughout each experiment, indicating that all PIV measurements were taken in the shear-thinning regime (i.e., not in the constantviscosity regions at high or low strain rates).

Finally, the variation in the angle $\delta$ with $R e$ is shown in Fig. 4(c). The Reynolds number is computed assuming that the oscillation frequency is given by the strain rate $(\Omega=\dot{\gamma})$. For all measurements, $\delta>45^{\circ}$, indicating that the flow is dominated by viscous effects.

\section{RESULTS}

\section{A. Time-averaged flow fields}

Vorticity fields were calculated from the measured mean velocity fields using least-squares differentiation and are presented in Fig. 5 for the four fluids at a range of $R e$. The fields are characterized by a series of vortices of alternating sign that are approximately evenly spaced along the entire Taylor-Couette flow cell. In each case, the magnitude of vorticity increases for $R e \gtrsim 150$, but the fields show little Re dependence for $R e \gtrsim 400$.

The most notable change between the Newtonian and shearthinning cases is the number of vortices formed; for the Newtonian case [Fig. 5(a)], there are six vortex pairs, leading to a mean wavelength of $\lambda / d \approx A R / 6 \approx 2.16$, while five pairs are present for all shear-thinning cases, with an increased wavelength of $\lambda / d \approx 2.59$. The tendency for shear thinning to cause an increase in the wavelength has also been noted in previous numerical, ${ }^{22}$ experimental, and analytical ${ }^{24}$ studies for large radius ratios.

In the Newtonian case, the positions of the vortices show little variation as $R e$ is increased, other than a slight widening of the vortices at either end of the flow cell. The positions and sizes of the vortices are more variable in the shear-thinning fluids. This can be seen most clearly in the most shear-thinning case [Fig. 5(d)], and will be discussed in more detail in Sec. III C.

Close examination of the vorticity fields for the Newtonian case indicate that the maxima and minima of vorticity do not occur at the center of the vortices, as might be expected, but tend to occur close to the vortex boundaries. This is particularly noticeable at higher Reynolds numbers ( $R e \gtrsim 400$ ). The tendency for vorticity to concentrate near the vortex boundaries is enhanced in the mildly shear-thinning case $[n=0.68$, Fig. 5 (b) ], where patches of reduced magnitude vorticity can be seen in the center of vortices at high Re. For the more strongly shear-thinning fluids [Figs. 5(c) and 5(d)], the vorticity tends to concentrate at a single vortex boundary, corresponding to the radial outward jet; this can be seen in Fig. 5 as the low vorticity lines between a positive vortex on the left and a negative vortex on the right.

In order to examine this trend in more detail, profiles of the mean vorticity at the center of the gap, $r=\left(r_{i}+r_{o}\right) / 2$, over a single vortex pair are shown in Fig. 6 . The wavelength was identified from the successive minima in the mean radial velocity profile along this line [shown in Figs. 6(a)-6(c)], and the vortex pair shown corresponds to the pair closest to the center of the test section (i.e., closest to $z / d=A R / 2=6.5$ ). The profiles across each wavelength are not always symmetric (or antisymmetric in the case of the vorticity) about $z / \lambda=0.5$ due to variations in the width of the individual vortices.

The profiles of the mean vorticity [Figs. 6(d)-6(f)] indicate that the largest magnitude vorticity occurs near the outward jets $(z / \lambda \approx 0.5)$ for all four fluids. For moderate Reynolds numbers $(R e \gtrsim 200)$, smaller magnitude peaks are present near the inward jets $(z / \lambda \approx 0.1$ and 0.9$)$ and local minima exist in the center of the vortices $(z / \lambda \approx 0.25$ and 0.75$)$. Within each vortex $(z / \lambda \approx 0.1-0.4$ and $\approx 0.6-0.9)$, the vorticity magnitude tends to be lower for the shear-thinning cases, compared to the Newtonian case.

The reduction in the vorticity in shear-thinning fluids is illustrated in Fig. 7, which shows the variation in the root-meansquare (rms) of vorticity in the meridian plane with the Reynolds number for each fluid. The rms value is not zero at very low Reynolds numbers before the onset of instability $(R e \lesssim 80)$ due to 


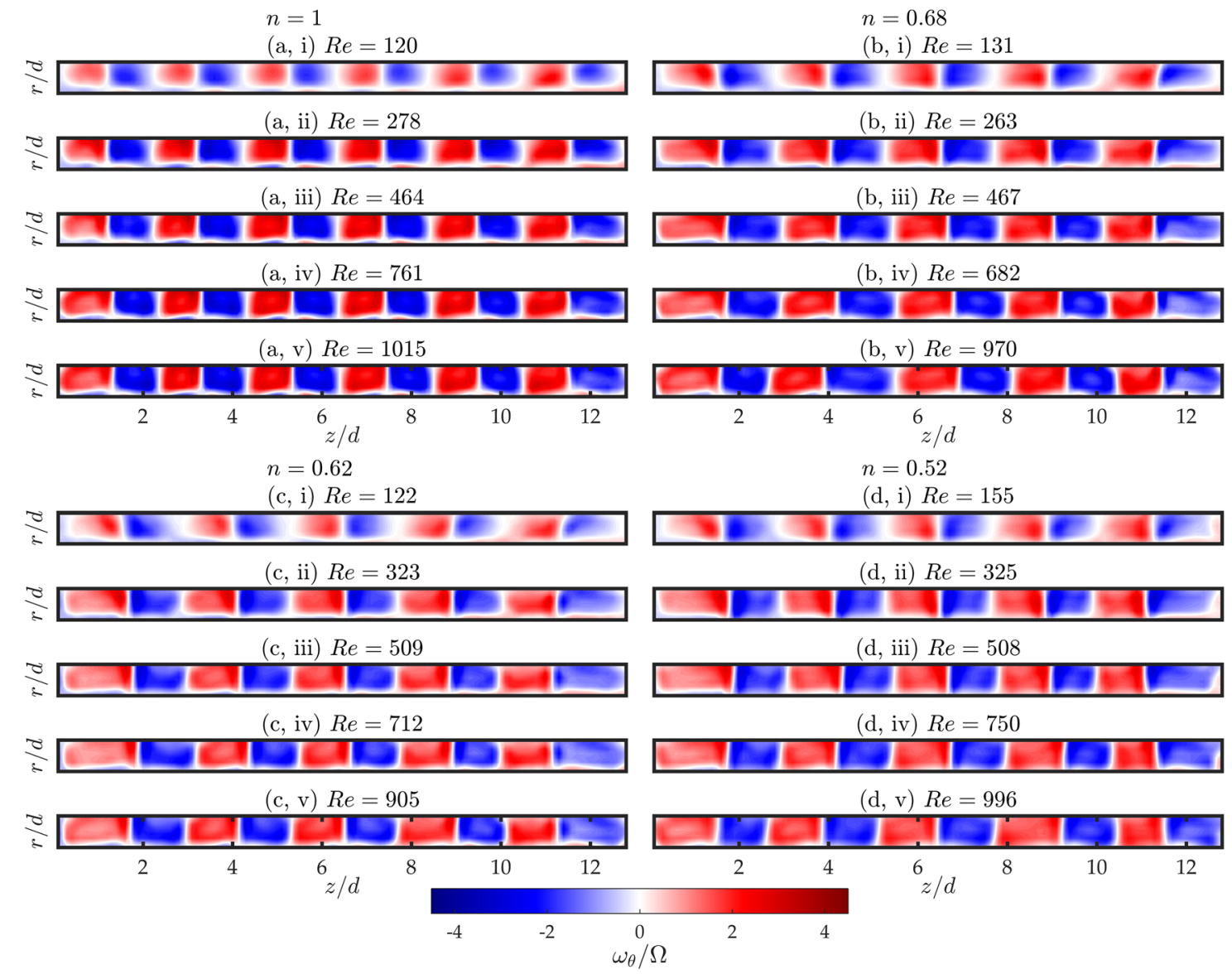

FIG. 5. Mean vorticity fields calculated for the Newtonian $[n=1$, (a)] and shear-thinning cases: $n=0.68$ (b); $n=0.62$, (c); and $n=0.52$, (d). Vorticity fields correspond to $R e \approx 150$ (i); $\approx 300$ (ii); $\approx 500$ (iii); $\approx 750$ (iv); and $\approx 1000$ (v).

the Ekman vortices at either end of the test section. ${ }^{34}$ The vorticity dramatically increases in the region $R e \approx 50-200$, before reaching a plateau by around $R e=600$. The decrease in the flow index (more shear thinning) is associated with a progressive reduction in the total magnitude of vorticity in the test section at high $R e$; by $R e \approx 1000$, the rms of vorticity for the most shear-thinning fluid $(n=0.52)$ is $70 \%$ of that found for the Newtonian case.

The changes in the size of vortices and the magnitude of vorticity are related to changes in the relative strength of the jets of fluid moving radially inward and outward. The outward jet transports fluid with high angular momentum from the inner cylinder into the center of the gap, creating an azimuthal jet that is thought to be responsible for the wavy instability. ${ }^{5,7}$ Thus the relative strength of the outward jet is an important factor in determining the onset of wavy vortex flow.

Figure 8 shows the variation in the strengths of the inward and outward jets $\left(u_{i}\right.$ and $u_{o}$, respectively), which are defined as the absolute value of the maximum and minimum radial velocity found from the mean velocity fields. As with the profiles of the mean azimuthal vorticity, the strength of the outward jet (closed symbols) increases sharply in the region $R e \approx 50-200$ for each fluid, before reaching a plateau. The strength of the outward jet shows very little dependence on the fluid rheology. The strength of the inward radial jet (open symbols) also increases in the region $R e \approx 50-200$ before reaching an approximately steady value for $R e \gtrsim 400$. However, unlike the outward jet, the strength of the inward jet depends on the flow rheology, with $u_{i}$ tending to be lower for shear-thinning fluids. This trend results in a large asymmetry in the magnitudes of the inward and outward jets [Fig. 8(b)] that is greatest for the most shear-thinning fluids shortly after the onset of the primary instability $(R e \approx 200-400)$. The asymmetry gradually converges toward the value seen in the Newtonian case, $\left(u_{o}-u_{i}\right) / \Omega d \approx 0.2$, as the Reynolds number is increased.

As the xanthan solutions are both shear thinning and weakly elastic, it is worth considering which rheological feature is responsible for the increased asymmetry with respect to the Newtonian case. Viscoelastic fluids in Taylor-Couette flow are known to undergo distinct instabilities at very low Reynolds number due to elastic forces, which result in the formation of diwhirls that are characterized by a strong and highly asymmetric radial jet. ${ }^{35}$ However, in 

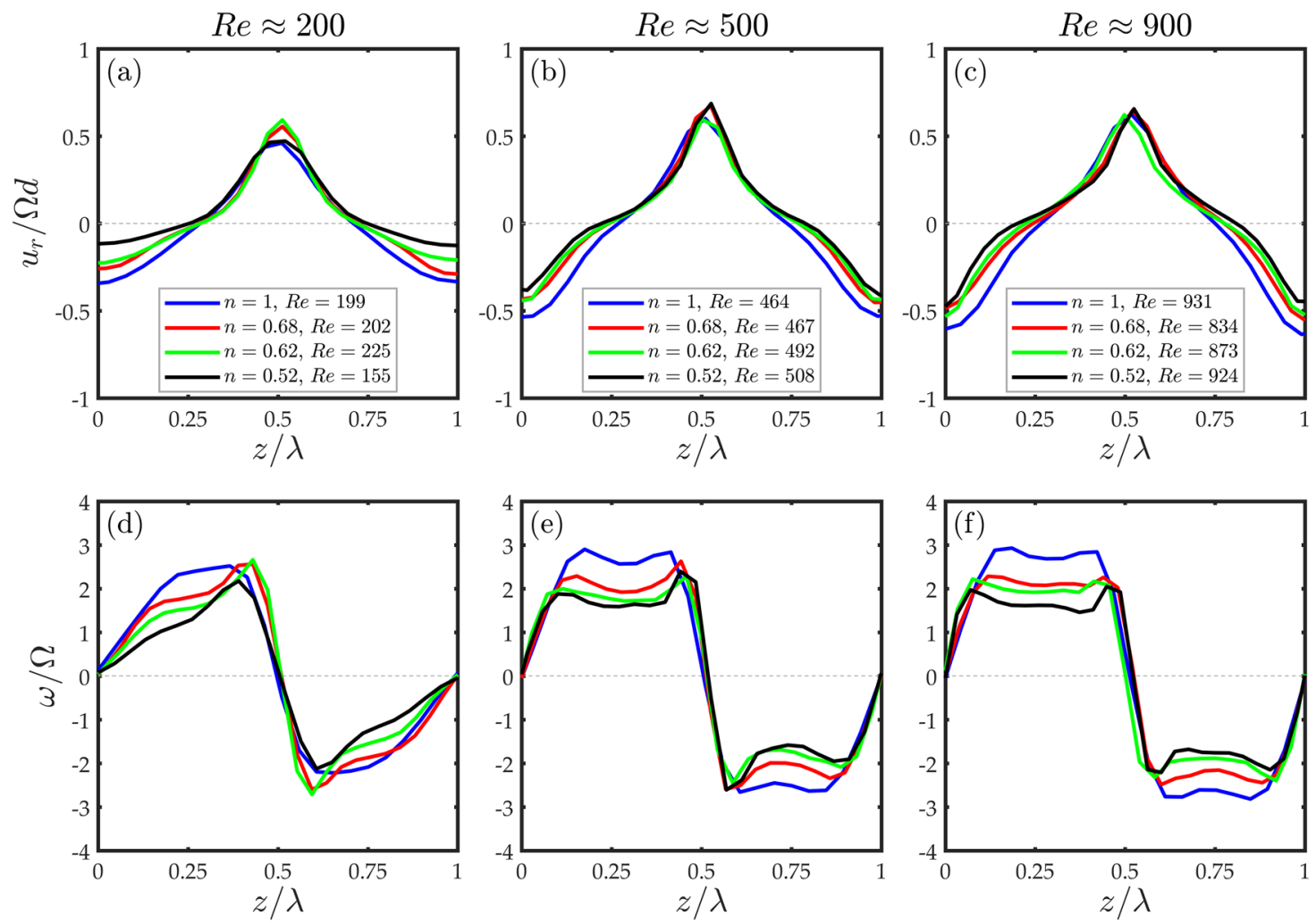

FIG. 6. Profiles of the mean radial velocity [top row, (a)-(c)] and mean vorticity [bottom row, (d)-(f)] at $r=\left(r_{i}+r_{0}\right) / 2$ along a single wavelength (i.e., for a single vortex pair). The wavelength shown corresponds to the vortex pair closest to the axial center of the test section. The Reynolds numbers shown are $\operatorname{Re} \approx 200$ [(a) and (d)], $R e \approx 500$ [(b) and (e)], and $R e \approx 900$ [(c) and (f)]. For $n=1$, the mean wavelength is $\lambda \approx A R / 6 \approx 2.16$ and for the shear-thinning fluids $\lambda \approx A R / 5 \approx 2.59$.

these diwhirls, the radial flow is significantly greater in the inward direction, ${ }^{35}$ in contrast to the trend seen in Fig. 8, where enhanced shear thinning and viscoelasticity are associated with a weakening of the inward jet. Therefore, the asymmetry observed in these

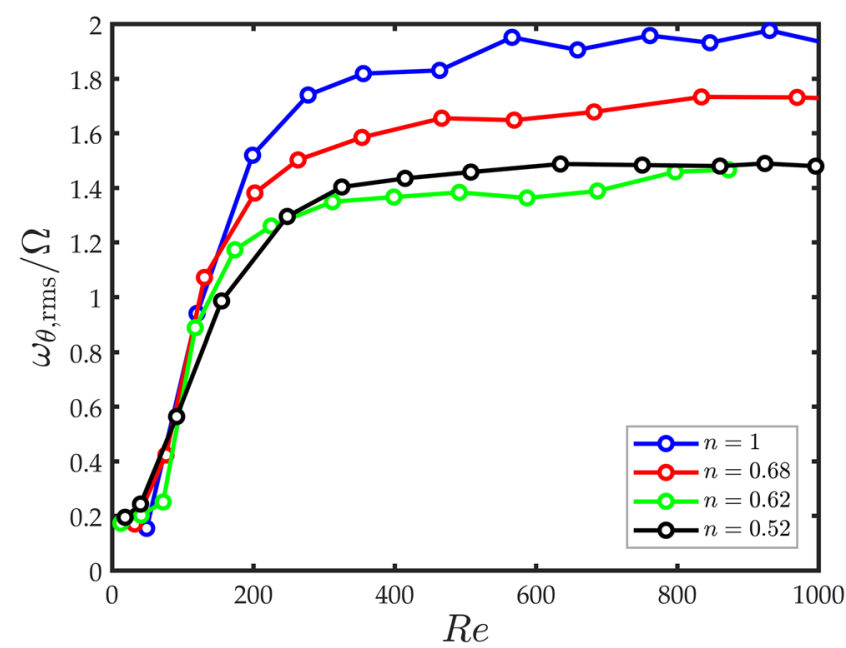

FIG. 7. Variation with Reynolds of the root-mean-square of vorticity, found from the mean velocity fields in the meridian plane, for four fluids of different flow indices. experiments can be attributed to the effects of shear thinning, rather than elasticity.

Some of the results observed here can be understood by considering Rayleigh-Bénard convection (thermal convection in a fluid layer enclosed by a hot surface below and a cold surface above), which is known to be a close analogy to Taylor-Couette flow ${ }^{1}$ (especially in the case of large $\eta$, where the effects of curvature are weak). In both cases, the instability is driven by an excess of a property at one boundary-thermal buoyancy in the case of RayleighBénard convection and angular momentum (which induce centrifugal forces) in the case of Taylor-Couette flow-with the unstable flow acting to redistribute that property. Following this analogy, a temperature-dependent viscosity in Rayleigh-Bénard convection is equivalent to a shear-thinning rheology in Taylor-Couette flow.

Temperature-dependent rheology is known to lead to an increase in the wavelength of thermal convection due to the reduced viscosity and high velocities in thermal upwellings, which can therefore redistribute the excess buoyancy from the lower boundary layer more efficiently compared to the isoviscous case. ${ }^{36-38}$ This also leads to an asymmetry in the flow, with the viscous downwelling regions having a reduced velocity and therefore being broader. ${ }^{3}$

In the case of Taylor-Couette flow of a shear-thinning fluid, the region of the excess property (i.e., angular momentum) also corresponds to a region of low viscosity (due to the high local shear rate), and the jets moving out of this region therefore have a high velocity 

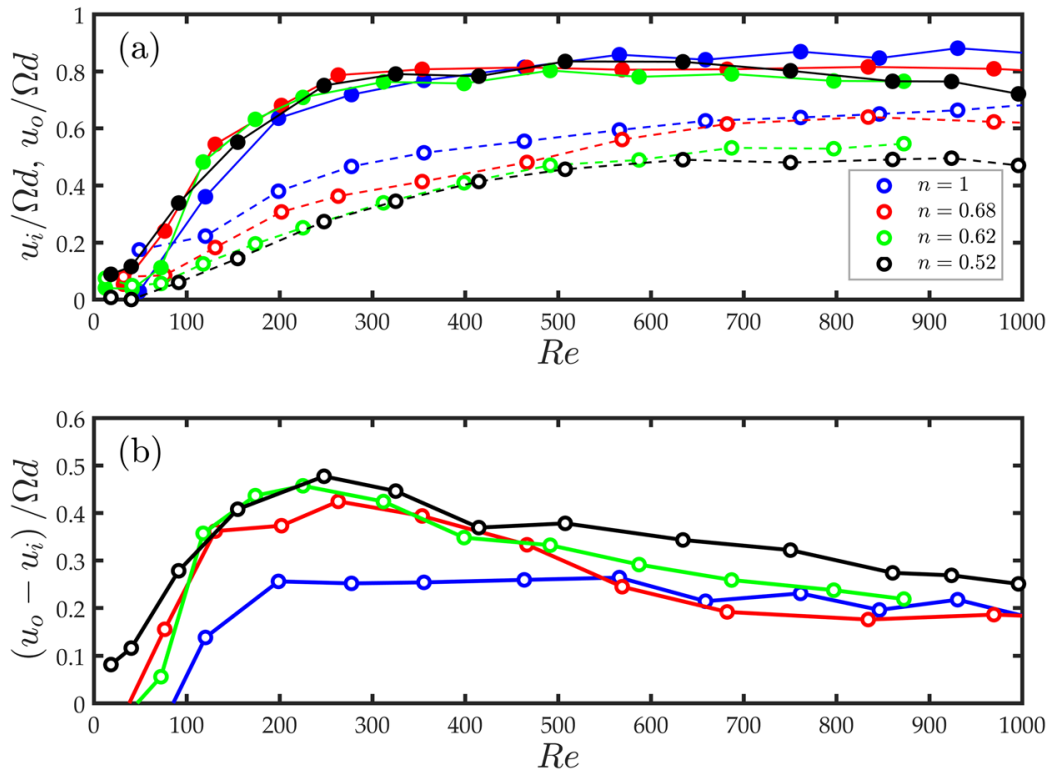

FIG. 8. Variation in the magnitude of the maximum radial velocity outward $\left(u_{0}\right.$, closed symbols and solid line) and inward ( $u_{i}$, open symbols and dashed line) (a). The difference between the two magnitudes is shown in (b). (see Fig. 8). This means that fewer jets are required to redistribute the angular momentum at the inner wall, which corresponds to an increase in the wavelength observed in Fig. 5 and in previous studies, ${ }^{22,24,25}$ and the asymmetry between the magnitude of the inward and outward jets. This asymmetry is manifested in the vorticity fields as a concentration of intense vorticity at the low viscosity outward jets [Fig. 5(d)].

\section{B. Flow transitions and unsteady dynamics}

Figure 9 shows the spatiotemporal maps acquired for each fluid as the Reynolds number was slowly increased. For low $R e$, the maps are characterized by a roughly uniform image intensity with white patches at the top and bottom of the vessel; this corresponds to Circular Couette Flow, with Ekman vortices at either end. As the critical Reynolds number is exceeded, a number of vortices are formed which can be identified from the horizontal light and dark bands. Despite a change in the wavelength, the overall structure of the flow seen in the spatiotemporal plots (and the vorticity fields shown in Fig. 5) for the Newtonian and shear-thinning fluids are remarkably similar. The shear-thinning maps do not show any signs of ribbon or spiral vortices or diwhirls that are associated with the elastic instability in viscoelastic fluids, ${ }^{35,39}$ indicating that the dynamics in the shear-thinning fluids are controlled by the same forces as in the Newtonian case (i.e., the dominance of centrifugal over viscous forces).

The critical Reynolds number, $R e_{c, 1}$, at which the transition from circular Couette flow to Taylor vortex flow occurs was estimated from the spatiotemporal maps, with an uncertainty of approximately \pm 3 , and the values are summarized in Table III. The critical Reynolds number is lower for all shear-thinning fluids relative to the Newtonian case, but the dependence of $R e_{c, 1}$ on the flow index in nonmonotonic. Cole ${ }^{34}$ examined the dependence of various flow features on the aspect ratio of the Taylor-Couette geometry, and found that $R e_{c, 1}$ was independent of the aspect ratio; therefore, the reduction in the critical Reynolds number for the shear-thinning fluids cannot be attributed to a change in the effective aspect ratio associated with the increase in wavelength in the shear-thinning cases.

The reduction in $R e_{c, 1}$ is consistent with previous experimental and numerical studies examining large radius ratio systems. ${ }^{22,24-26}$ Bahrani et al. ${ }^{27}$ used linear stability analysis to estimate $R e_{c, 1}$ for various shear-thinning fluids in a small radius ratio system $(\eta=0.4)$ and found that it varied nonmonotonically with flow index, reaching a minimum at around $n=0.65$. Their predictions were supported by experimental measurements and are consistent with the observation in the current experiments that shear thinning does not have a monotonic effect on the critical Reynolds number.

For the Newtonian fluid [Fig. 9(a)], the vortices form at $R e=102$, which is very close to the value predicted by Esser and Grossmann $^{40}$ for this radius ratio (103.9). Once the flow has transitioned, the spatiotemporal map does not show any clear changes as the Reynolds number is increased up to $\approx 1000$. In contrast, the positions of the vortices in the shear thinning exhibit slow drifts in their size and position for $R e \gtrsim 500$. This variability was also observed in the time-averaged vorticity fields (Fig. 5) and will be explored in Sec. III C.

In order to study the higher order instabilities and estimate the critical Reynolds number for the transition to Wavy Vortex Flow, $R e_{c, 2}$, it is necessary to examine the frequency spectra of the spatiotemporal maps. In order to do this, the maps were divided into segments of 256 data points (with $50 \%$ overlap between segments) and the frequency spectra at each axial position were computed using the Fast Fourier Transform (FFT) and averaged to form stacks. These are referred to as frequency-Reynolds number maps and are shown in Fig. 10 for all four fluids, where the vertical axis is now frequency and the dark regions represent peaks in energy content.

The frequency-Re maps always contain peaks at the rotational speed (indicated by the red line) and its harmonics, due to small 
(a) $n=1$

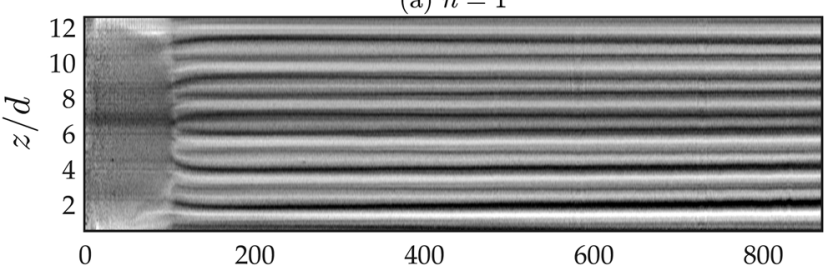

(b) $n=0.68$

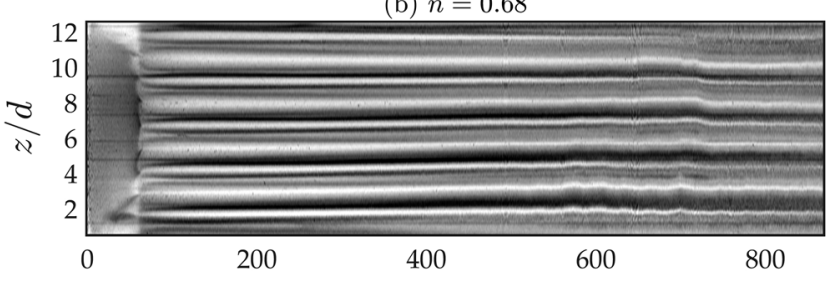

(c) $n=0.62$

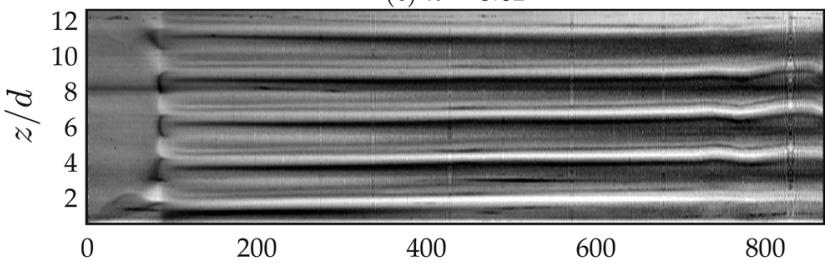

(d) $n=0.52$

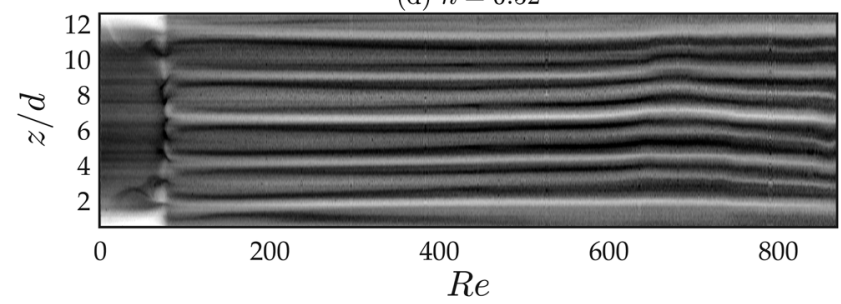

FIG. 9. Spatiotemporal maps produced using mica flakes, for four different fluids, including a Newtonian case, $n=1$ (a), and three shear-thinning cases $n=0.68(\mathrm{~b})$, $n=0.62(\mathrm{c})$, and $n=0.52$ (d).

vibrations in the experimental system caused by the rotation of the motor. The harmonics can quickly exceed the Nyquist frequency (which correspond to the top of the $y$-axis), which introduces aliasing and causes the ridges to appear to bounce off the top and bottom of the maps. For the shear-thinning fluids, Re does not depend linearly on the rotational speed, causing the ridges to curve slightly, which becomes more noticeable when the flow index is small.

TABLE III. Critical Reynolds number of Taylor vortex flow $\left(R e_{c, 1}\right)$ and wavy vortex flow $\left(R e_{c, 2}\right)$ and the instability frequency in wavy flow.

\begin{tabular}{lccc}
\hline \hline$n$ & $R e_{c, 1}$ & $R e_{c, 2}$ & $f_{W V F}\left(\frac{2 \pi}{\Omega}\right)$ \\
\hline 1 & 106 & 825 & 1.14 \\
0.68 & 61 & 826 & 0.878 \\
0.62 & 83 & $>874$ & $\mathrm{n} / \mathrm{a}$ \\
0.52 & 77 & 664 & 0.881 \\
\hline \hline
\end{tabular}

(a) $n=1$

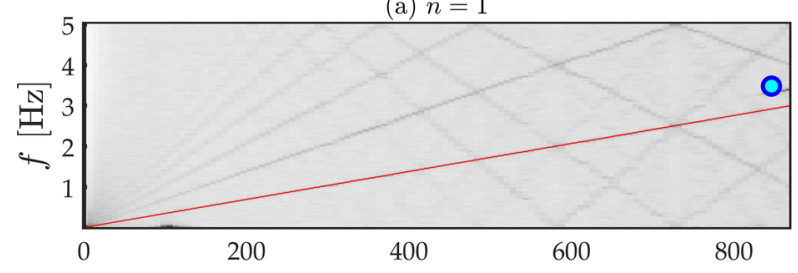

(b) $n=0.68$

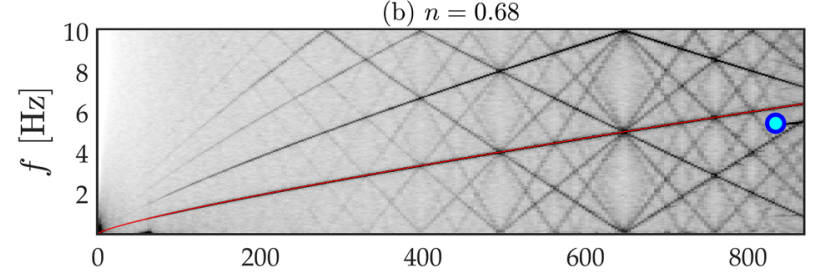

(c) $n=0.62$

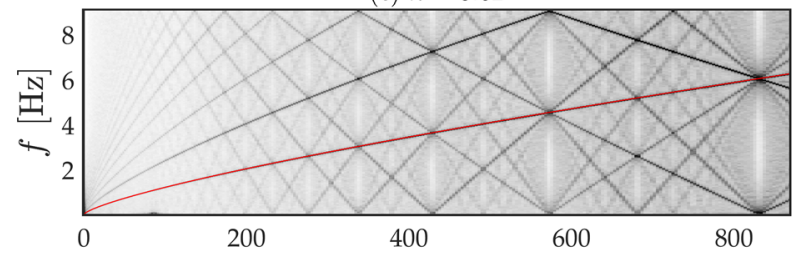

(d) $n=0.52$

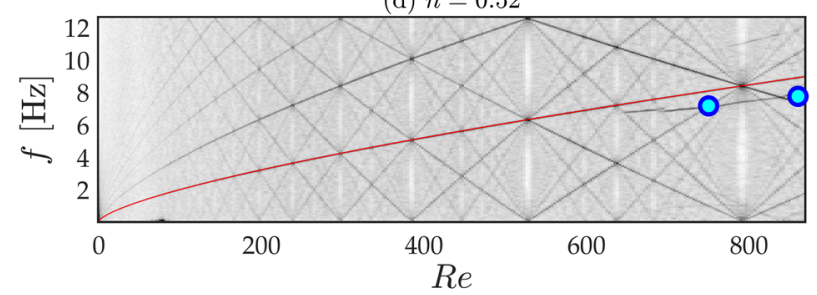

FIG. 10. Frequency-Reynolds number maps produced using mica flakes for four different fluids, including a Newtonian case, $n=1$ (a), and three shear-thinning cases $n=0.68$ (b), $n=0.62$ (c), and $n=0.52$ (d). The scale is arbitrary, with dark regions corresponding to elevated FFT amplitudes. The blue circles denote the frequencies of the wavy instability found from the PIV measurements of the same fluid (PIV measurements in which no instability was observed are not shown), indicating good agreement between the PIV and flow visualization measurements.

The frequency-Re map for the Newtonian case shows little change with Reynolds number until $R e \approx 810$, when a new ridge appears slightly above the red line, which corresponds to the onset of the wavy instability, occurring at a slightly higher frequency than the rotational speed $(3-4.5 \mathrm{~Hz})$. The PIV measurements in this Re range also indicate a wavy instability at this frequency, demonstrating the consistency between the PIV and flow visualization measurements and the reproducibility of the flow dynamics, once the same protocol is employed.

It should be noted that the critical Reynolds number for the onset of the wavy instability, 810 , is relatively high compared to other experiments in the literature. ${ }^{2}$ This is likely to be a consequence of the moderate aspect ratio of our system, as $R e_{c, 2}$ is known to increase sharply at low AR. ${ }^{34}$

The flow index has a nonmonotonic effect on the critical Re for the onset of waviness, as can be seen in Fig. 10 and Table III. 

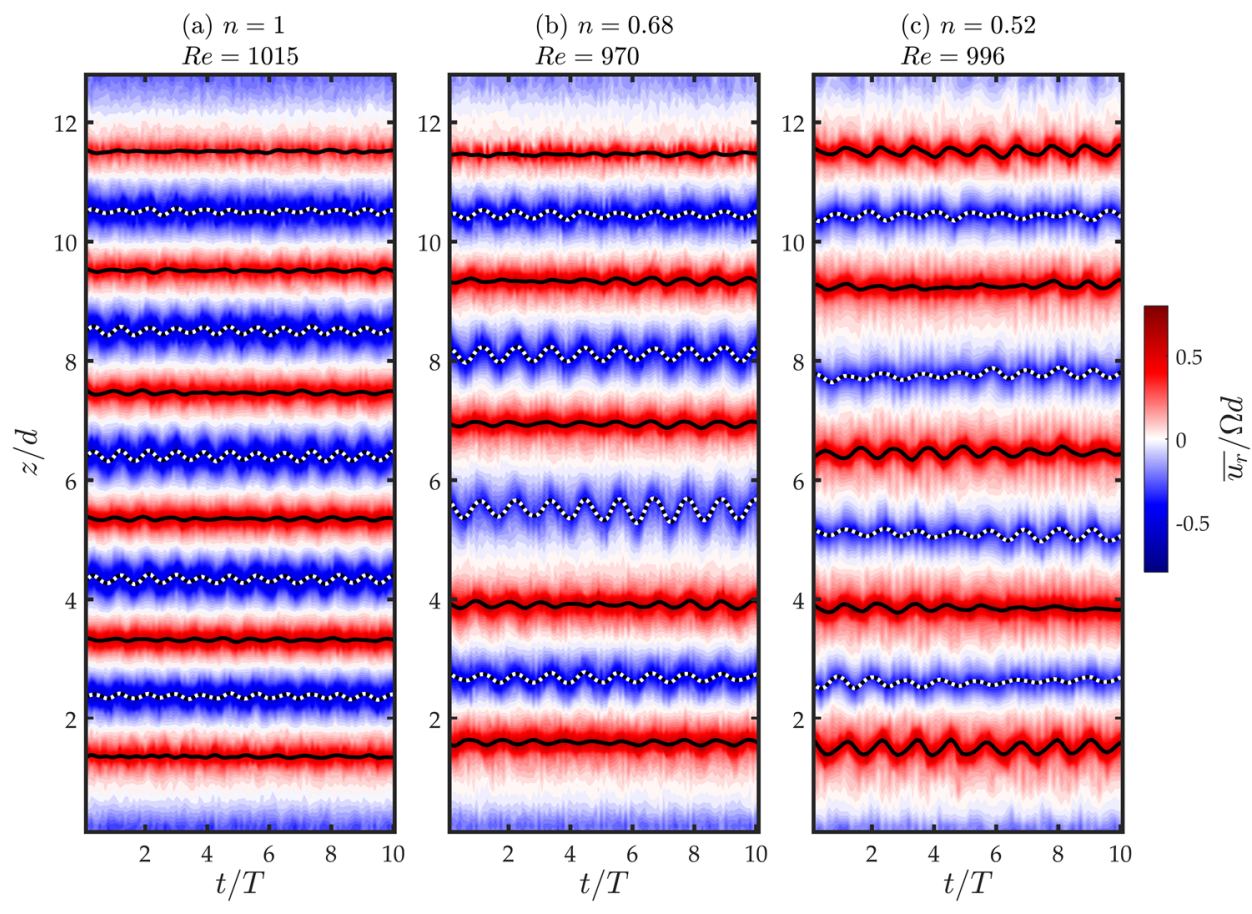

FIG. 11. Variation in the radial velocity, averaged across the gap, with time and axial position at $R e \sim 1000$ for a Newtonian fluid (a), and two shear-thinning fluids, $n=0.68$ (b) and $n=0.52$ (c). The solid black and dashed lines represent the positions of the outward and inward jets, respectively, which were identified from the local maxima and minima in the radial velocity.
For the most-shear-thinning case, $R e_{c, 2}$ is reduced to 660 , but for the intermediate cases $(n=0.62$ and 0.68$), R e_{c, 2}$ is larger than that found for the Newtonian fluid; in fact, it is raised to such an extent for $n=0.62$ that the instability is not observed throughout the entire $R e$ range examined, i.e., $R e_{c, 2}>992$.

For the Newtonian fluid, the wavy instability frequency scales as $f_{W V F}=1.14 \Omega / 2 \pi$, whereas for the shear-thinning cases that exhibit wavy flow, the instability frequency is lower than the rotational frequency, with $f_{W V F} \approx 0.88 \Omega / 2 \pi$ in both cases (Table III). This represents an inversion of the scaling constant (i.e., $1.14^{-1} \approx 0.88$ ) and indicates that the time scales of the wavy flow will be larger for the shear-thinning fluids.

The wavy instability can be studied in further detail by examining the temporal variations in the PIV measurements of the radial velocity, as shown in Fig. 11, for the three fluids in which WVF was observed. In order to reduce the dimensions of the PIV measurements and clearly identify the motion of the various jets, the velocity fields were averaged in the radial direction. The solid black lines correspond to local maxima of the outward jets and the dashed black and white lines correspond to the maxima of the inward jets.

For the Newtonian case [Fig. 11(a)], the positions of the inward jets clearly oscillate at an apparently constant frequency in the axial direction, while the outward jets remain relatively fixed. The reduced amplitude of the waviness of the outward jet, $\delta_{z, o}$ (i.e., the amplitude of its maximum axial displacement, indicated by the black lines in Fig. 11) relative to that of the inward jet, $\delta_{z, i}$, was also observed by Wereley and Lueptow, ${ }^{4}$ Imomoh, Dusting, and Balabani ${ }^{29}$ and Nemri et al. ${ }^{41}$ The variation in $\delta_{z, i}$ and $\delta_{z, o}$ along the cylinder length is shown in Fig. 12. The outward jets oscillate at a constant amplitude of $\delta_{z, o} / d \approx 0.02$, while the inward jets have a variable amplitude that reaches a maximum of $\delta_{z, i} / d \approx 0.08$ at the center of the flow cell.
The radial velocity maps found for the shear-thinning fluids, shown in Figs. 11(b) and 11(c), exhibit several differences from the Newtonian case. The most noticeable difference is that the wavy instability is now clearly apparent in both the inward and the outward jets, e.g., at $z / d \approx 4$ in Figs. 11 (b) and 11 (c). This can also be seen in Fig. 12, where for the most shear-thinning case $(n=0.52)$, the amplitudes of the waves at the outward jets for $z / d>6$ exceed those of the waves at the inward jets.

It is interesting to note that shear thinning is associated with a decrease in the magnitude of the inward jet and has little effect on the magnitude of the outward jets, as was discussed with respect
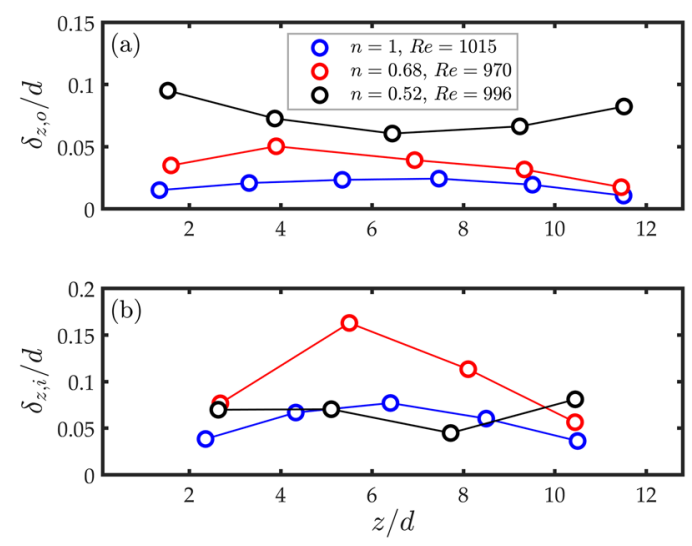

FIG. 12. Variation in the amplitude of the waves at the outward (a) and inward (b) jets for three fluids in which wavy vortex flow was observed; Newtonian, $n=1$, $n=0.68$, and $n=0.52$. 
to Fig. 8; therefore, it is slightly surprising that the shear-thinning fluids are associated with an increase in the amplitude of the waves at these points. This suggests that the amplitude of the instability is not dependent on the strength of the jets that are thought to drive it.

\section{Vortex drift}

Figure 12 indicates that while the wave amplitudes in a Newtonian fluid are either constant or smoothly varying (at the inward and outward jets, respectively), those in a shear-thinning fluid are highly variable along the spanwise direction.

The tendency for spatial variability in the amplitude of the wavy instability in shear-thinning fluids is consistent with the previous observation that shear thinning is associated with spatial variations in the size of vortices. Considering the time-averaged vorticity fields shown in Fig. 5, it was previously noted that the positions of the vortices in the Newtonian fluid [Fig. 5(a)] show little variation as $R e$ is increased, other than a slight increase in the size of the vortices at either end of the flow cell. However, this is not the case for the shear-tinning fluids. The most weakly shear-thinning case $[n=0.68$, Fig. 5(b)] shows a general elongation of the vortices in the lower half of the test section $(z / d \lesssim 6)$ and a contraction of the vortices near the other end. Changes in the vortex spacing are also observed for the more shear-thinning cases $[n=0.62$ and 0.52 , shown in Figs. 5 (c) and 5(d)], although in these cases the drift in the position of individual vortices is not monotonic or consistent.

The phenomenon of this drift, i.e., the gradual contraction or elongation of vortices, can also be seen in the spatiotemporal maps shown in Fig. 9. For the Newtonian case, once the vortices form at
$R e_{c, 1}$, their axial positions remain essentially constant over the entire Reynolds number range examined, as was observed for the PIV measurements. In contrast, for the shear-thinning fluids, the vortices exhibit a gradual drift in position. This can most clearly be seen at $R e \approx 850$ in Fig. 9(c) and $R e \approx 700$ in Fig. 9(d). These shifts in the position of vortices occur over long time periods (of the order of minutes, or several hundred cylinder revolutions) and are therefore not related to the wavy instability, which occurs over much shorter time scales.

The variation in the vortex spacing can be quantified using the time-averaged velocity fields. The boundaries between vortices can be identified from the points where the vorticity (averaged in the radial direction) is zero. The variation in these boundaries with $R e$ for each fluid is shown in Fig. 13. In agreement with the flow visualization data, Fig. 13 shows that the vortex spacing is independent of $R e$ for the Newtonian fluid, but exhibits low-frequency variations for $R e \gtrsim 500$ for the shear-thinning fluids.

The variability can be quantified using the parameter, $\Delta W_{v} / \overline{W_{v}}$, where $\Delta W_{v}$ is the difference between the maximum and minimum vortex axial length at a given $R e$ and $\overline{W_{v}}$ is the mean vortex axial length. The vortices at either end of the flow cell were neglected in order to reduce the influence of end effects.

The variation of $\Delta W_{v} / \overline{W_{v}}$ with the Reynolds number for each fluid is shown in Fig. 14. In all cases, there is considerable spatial variability when the vortices initially form near $R e_{c, 1}$, after which $\Delta W_{v} / \overline{W_{v}}$ decreases to $\approx 0.13$. The trends show some scatter, which is a consequence of the relatively small number of vortices in each measurement. However, it is clear that in the Newtonian case, the variability parameter remains close to 0.13 , while for the shear-thinning fluids it increases with $R e$. The variability is largest in the most
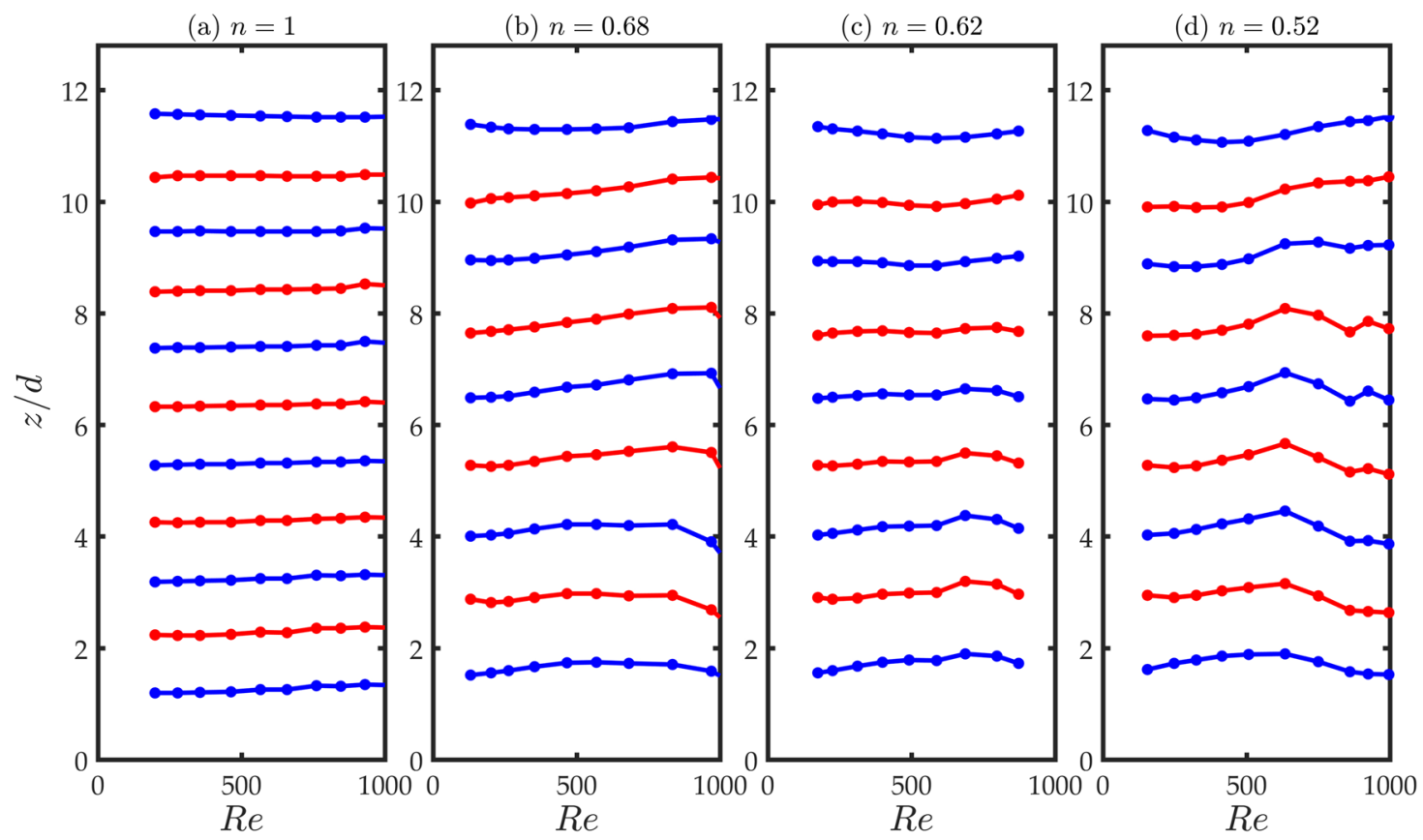

FIG. 13. Variation in the positions of vortex boundaries with Re for the Newtonian (a) and shear-thinning fluids [(b) and (d)]. Blue and red lines correspond to the boundaries at outward and inward jets, respectively. 


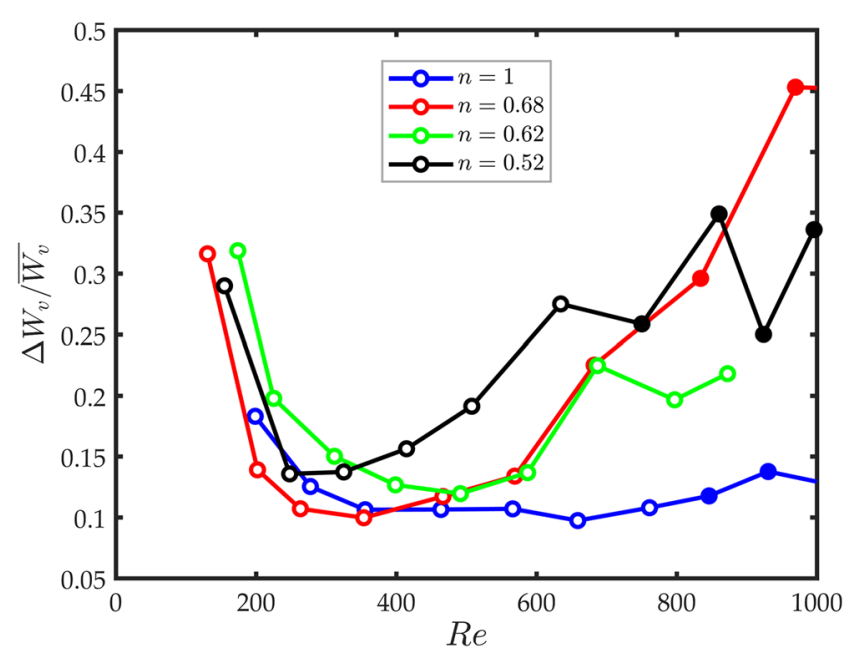

FIG. 14. Variability of the axial length of vortices as a function of Reynolds number for each of the four fluids. The variability is defined as the difference between the maximum and the minimum vortex widths at a given $R e$, divided by the mean vortex width (neglecting the end vortices).

shear-thinning fluid, clearly demonstrating the correlation between non-Newtonian rheology and the tendency for vortices to exhibit low frequency drifts in their size and position.

The drift is not observed for $n=0.62$ for $R e \approx 400$, where $\delta \approx 50^{\circ}$ [Fig. 4(c)], but is observed for all shear-thinning fluids (for $R e \gtrsim 500)$, even in the case of $\mathrm{n}=0.68$ when the fluid is less viscoelastic $\left(\delta \approx 70^{\circ}\right)$, which suggests that the drift is not a consequence of viscoelasticity. The absence of other flow features associated with an elastic-controlled instabilities (e.g., ribbon and spiral vortices ${ }^{39}$ ) when drift is observed also indicates that viscoelasticity is not the dominant rheological feature controlling the drift.

This drift was also observed in the laser-Doppler anemometry measurements of Escudier, Gouldson, and Jones, ${ }^{25}$ for a shearthinning fluid and a second fluid which was also thixotropic and viscoelastic. They did not observe any drift for the Newtonian reference case, in agreement with our results and confirming that the drift is a consequence of the non-Newtonian rheology. They reported a constant drift velocity of $3-4 \mu \mathrm{m} / \mathrm{s}$ (for a gap of $49.6 \mathrm{~mm}$ and $\eta=0.506$ ), and noted that the direction of drift was different for the purely shear-thinning fluid and the thixotropic and viscoelastic case, which they attributed to the additional rheological phenomena. However, it is clear that drift in a single direction cannot continue indefinitely, as this would eventually lead to the removal of all but one of the vortices. Therefore, it is more plausible that the drift is random and the direction can vary with time and Reynolds number, as can be seen in the flow-visualization measurements in Fig. 9(d).

In terms of the stability of the system, the tendency for the width of individual vortices to vary both with Reynolds number and along the span of the fluid domain indicates that for the shearthinning flows, there exists a continuous spectrum of stable axial wavelengths, while for the Newtonian case the flow is characterized by a single wavelength. This suggests that the analytical predictions of the axial wavelength for shear-thinning fluids should be treated with caution, as the flow may not be characterized by a single value. The performance of analytical predictions is also likely to be complicated by the moderate aspect ratio employed in this study, which is likely to restrict the drift of the position and size of vortices and limit the range of permissible wavelengths.

\section{CONCLUSIONS}

We performed a series of experiments examining TaylorCouette flow of a Newtonian and three shear-thinning fluids, in which the Reynolds number was slowly increased, such that the circular Couette flow, Taylor vortex flow and wavy vortex flow regimes were observed. The flow dynamics were examined using two complementary processes, particle-image velocimetry, to measure the velocity field in the meridian plane over short time periods, and flow visualization to continuously monitor the flow and observe transitions between regimes.

The time-averaged vorticity fields indicated that shear thinning was associated with an increase in the axial wavelength of Taylor vortex flow and caused the vorticity to concentrate along the vortex boundaries-especially at the outward jets-rather than at the vortex cores. Shear thinning also coincides with a reduction in the root-mean-square of the vorticity in the meridian plane and in the strength of the inward jets, despite having no significant effects on the strength of the outward jets.

The critical Reynolds numbers for transition to Taylor vortex flow and wavy vortex flow both showed a nonmonotonic dependence on the flow index; the critical Reynolds number for the onset of TVF was always lower for the non-Newtonian fluids, but there was considerable variation in the critical Re for the transition to WVF. Once the wavy instability was established, shear thinning was associated with increases in the amplitudes of the traveling waves at the inward and outward jets.

Finally, the Taylor vortices in the shear-thinning fluids were found to exhibit low-frequency changes in their size and position, over a time scale of several hundred cylinder rotations, which was not related to wavy instability. This drift was not observed for the Newtonian fluid and was most significant in the most shear-thinning fluid examined.

\section{ACKNOWLEDGMENTS}

Financial support for this work from the Engineering and Physical Sciences Research Council (EPSRC) Manufacturing the Future programme (No. EP/N024915/1) is gratefully acknowledged.

\section{REFERENCES}

${ }^{1}$ S. Grossmann, D. Lohse, and C. Sun, "High-Reynolds number Taylor-Couette turbulence," Annu. Rev. Fluid Mech. 48, 53-80 (2016).

${ }^{2}$ M. A. Fardin, C. Perge, and N. Taberlet, "The hydrogen atom of fluid dynamics'-Introduction to the Taylor-Couette flow for soft matter scientists," Soft Matter 10, 3523-3535 (2014).

${ }^{3}$ C. D. Andereck, S. S. Liu, and H. L. Swinney, "Flow regimes in a circular Couette system with independently rotating cylinders," J. Fluid Mech. 164, 155-183 (1986).

${ }^{4}$ S. Wereley and R. M. Lueptow, "Spatio-temporal character of non-wavy and wavy Taylor-Couette flow,” J. Fluid Mech. 364, 59-80 (1998).

${ }^{\mathbf{5}}$ A. Akonur and R. M. Lueptow, "Three-dimensional velocity field for wavy Taylor-Couette flow," Phys. Fluids 15, 947 (2003). 
${ }^{6}$ C. A. Jones, “The transition to wavy Taylor vortices," J. Fluid Mech. 157, 135-162 (1985).

${ }^{7}$ K. T. Coughlin and P. S. Marcus, "Modulated waves in Taylor-Couette flow Part 2. Numerical simulation," J. Fluid Mech. 234, 19-46 (1992).

${ }^{8}$ C. S. Dutcher and S. J. Muller, "Spatio-temporal mode dynamics and higher order transitions in high aspect ratio Newtonian Taylor-Couette flows," J. Fluid Mech. 641, 85-113 (2009).

${ }^{9}$ U. B. Holeschovsky and C. L. Cooney, "Quantitative description of ultrafiltration in a rotating filtration device," AIChE J. 37, 1219-1226 (1991).

${ }^{10}$ E. K. Hill, B. Krebs, D. G. Goodall, G. J. Howlett, and D. E. Dunstan, "Shear flow induces amyloid fibril formation," Biomacromolecules 7, 10-13 (2006).

${ }^{11}$ G. A. Ameer, E. A. Grovender, B. Obradovic, C. L. Cooney, and R. Langer, "RTD analysis of a novel Taylor-Couette flow device for blood detoxification," AIChE J. 45, 633-638 (1999).

${ }^{12}$ S. Vedantam and J. B. Joshi, "Annular centrifugal contactors-A review," Chem. Eng. Res. Des. 84, 522-542 (2006).

${ }^{13}$ B. Haut, H. Ben Amor, L. Coulon, A. Jacquet, and A. Halloin, "Hydrodynamics and mass transfer in a Couette-Taylor bioreactor for the culture of animal cells," Chem. Eng. Sci. 58, 777-784 (2003).

${ }^{14}$ S. J. Curran and R. A. Black, "Quantitative experimental study of shear stresses and mixing in progressive flow regimes within annular-flow bioreactors," Chem. Eng. Sci. 59, 5859-5868 (2004).

${ }^{15}$ B. Kong, J. V. Shanks, and R. D. Vigil, "Enhanced algal growth rate in a Taylor vortex reactor," Biotechnol. Bioeng. 110, 2140-2149 (2013).

${ }^{16} \mathrm{~N}$. Ashrafi, "Stability analysis of shear-thinning flow between rotating cylinders," Appl. Math. Modell. 35, 4407-4423 (2011).

${ }^{17}$ S. Mueller, E. W. Llewellin, and H. M. Mader, "The rheology of suspensions of solid particles," Proc. R. Soc. London, Ser. A 466, 1201-1228 (2010).

${ }^{18}$ P. Coussot, "Yield stress fluid flows: A review of experimental data," J. NonNewtonian Fluid Mech. 211, 31-49 (2014).

${ }^{19}$ N. Cagney, T. Zhang, R. Bransgrove, M. J. Allen, and S. Balabani, "Effects of cell motility and morphology on the rheology of algae suspensions," J. Appl. Phycol. 29, 1145-1157 (2017).

${ }^{20} \mathrm{M}$. M. Cross, "Relation between viscoelasticity and shear-thinning behaviour in liquids," Rheol. Acta 18, 609-614 (1979).

${ }^{21}$ O. Coronado-Matutti, P. R. Souza Mendes, and M. S. Carvalho, "Instability of inelastic shear-thinning liquids in a Couette flow between concentric cylinders," J. Fluids Eng. 126, 385-390 (2004).

${ }^{22}$ T. J. Lockett, S. M. Richardson, and W. J. Worraker, "The stability of inelastic non-Newtonian fluids in Couette flow between concentric cylinders: A finite-element study," J. Non-Newtonian Fluid Mech. 43, 165-177 (1992).

${ }^{23}$ S. Khali, R. Nebbali, and K. Bouhadef, "Numerical investigation of non-Newtonian fluids flows between two rotating cylinders using latticeBoltzmann method," Int. Scholarly Sci. Res. Innovation 7, 1999-2005 (2013).
${ }^{24}$ B. Alibenyahia, C. Lemaitre, C. Nouar, and N. Ait-Messaoudene, "Revisiting the stability of circular Couette flow of shear-thinning fluids," J. Non-Newtonian Fluid Mech. 183-184, 37-51 (2012).

${ }^{25}$ M. P. Escudier, I. W. Gouldson, and D. M. Jones, "Taylor vortices in Newtonian and shear-thinning liquids," Proc. R. Soc. London, Ser. A 449, 155-175 (1995).

${ }^{26}$ V. Sinevic, R. Kuboi, and A. W. Nienow, "Power numbers, Taylor numbers and Taylor vortices in viscous Newtonian and non-Newtonian fluids," Chem. Eng. Sci. 41, 2915-2923 (1986).

${ }^{27}$ S. A. Bahrani, C. Nouar, A. Neveu, and S. Becker, "Transition to chaotic TaylorCouette flow in shear-thinning fluids," in 22ème Congrès Français de Mécanique, Lyon, 2015.

${ }^{28}$ J. Dusting and S. Balabani, "Mixing in a Taylor-Couette reactor in the non-wavy flow regime," Chem. Eng. Sci. 64, 3103-3111 (2009).

${ }^{29} \mathrm{E}$. Imomoh, J. Dusting, and S. Balabani, "On the quasiperiodic state in a moderate aspect ratio Taylor-Couette flow," Phys. Fluids 22, 044103 (2010).

${ }^{30} \mathrm{Q}$. Xiao, T. T. Lim, and Y. T. Chew, "Effect of acceleration on the wavy Taylor vortex flow," Exp. Fluids 32, 639-644 (2002).

${ }^{31}$ N. Abcha, N. Latrache, F. Dumouchel, and I. Mutabazi, "Qualitative relation between reflected light intensity by Kalliroscope flakes and velocity field in the Couette-Taylor flow system," Exp. Fluids 45, 85-94 (2008).

${ }^{32}$ B. Martínez-Arias, J. Peixinho, O. Crumeyrolle, and I. Mutabazi, "Effect of the number of vortices on the torque scaling in Taylor-Couette flow," J. Fluid Mech. 748, 756-767 (2014).

${ }^{33}$ M. V. Majji, S. Banerjee, and J. F. Morris, "Inertial flow transitions of a suspension in Taylor-Couette geometry," J. Fluid Mech. 835, 936-969 (2018).

${ }^{34}$ J. A. Cole, "Taylor-vortex instability and annulus-length effects," J. Fluid Mech. 75, 1-15 (1976).

${ }^{35}$ A. Groisman and V. Steinberg, "Solitary vortex pairs in viscoelastic Couette flow," Phys. Rev. Lett. 78, 1460-1463 (1997).

${ }^{36}$ H. C. Nataf and F. M. Richter, "Convection experiments in fluids with highly temperature-dependent viscosity and the thermal evolution of the planets," Phys. Earth Planet. Inter. 29, 320-329 (1982).

${ }^{37} \mathrm{P}$. J. Tackley, "Effects of strongly variable viscosity on three-dimensional compressible convection in planetary mantles," J. Geophys. Res.: Solid Earth 101, 3311-3331, https://doi.org/10.1029/95jb03211 (1996).

${ }^{38}$ S. Zhong, M. T. Zuber, L. Moresi, and M. Gurnis, "Role of temperaturedependent viscosity and surface plates in spherical shell models of mantle convection," J. Geophys. Res.: Solid Earth 105, 11063-11082, https://doi.org/10.1029/2000jb900003 (2000).

${ }^{39}$ B. M. Baumert and S. J. Muller, "Axisymmetric and non-axisymmetric elastic and inertio-elastic instabilities in Taylor-Couette flow," J. Non-Newtonian Fluid Mech. 83, 33-69 (1999).

${ }^{40} \mathrm{~A}$. Esser and S. Grossmann, "Analytic expression for Taylor-Couette stability boundary," Phys. Fluids 8, 1814-1819 (1998).

${ }^{41}$ M. Nemri, S. Cazin, S. Charton, and E. Climent, "Experimental investigation of mixing and axial dispersion in Taylor-Couette flow patterns," Exp. Fluids 55, 1769-1784 (2014). 